\title{
Understanding host response to infectious salmon anaemia virus in an Atlantic salmon cell line using single-cell RNA sequencing
}

Ophélie Gervais ${ }^{1}$, Remi Gratacap ${ }^{1}$, Athina Papadopoulou ${ }^{1}$, Ross D. Houston ${ }^{1}$, Musa A. Hassan ${ }^{1}$, Diego Robledo ${ }^{1}$

${ }^{1}$ The Roslin Institute and Royal (Dick) School of Veterinary Studies, University of Edinburgh, Midlothian EH25 9RG, UK

\section{Abstract}

Background: Infectious Salmon Anaemia Virus (ISAV) is an Orthomixovirus that currently represents a large problem for salmonid aquaculture worldwide. Prevention and treatment methods are only partially effective. Genetic selection and genome engineering strategies have potential to develop ISAV resistant salmon stocks. However, this requires a detailed understanding of the genomic regulation of ISAV pathogenesis. Here, we used single cell RNA sequencing on a salmonid cell line to provide a high dimensional insight into the transcriptional landscape that underpin host-virus interactions during ISAV infection at the single cell level.

Results: Salmon head kidney 1 (SHK-1) cells were single-cell RNA sequenced before challenge, and at $24 \mathrm{~h}, 48 \mathrm{~h}$, and $96 \mathrm{~h}$ post-ISAV challenge. The results revealed marked changes in the host transcriptome at $48 \mathrm{~h}$ and $96 \mathrm{~h}$ post-infection, even in uninfected cells, potentially suggesting paracrine signalling. This paracrine activation of uninfected cells seemed to be unspecific, involving pathways such as mRNA sensing, ubiquitination or proteasome, and also the up-regulation of the mitochondrial ribosome genes. At $24 \mathrm{~h}$ post infection, cells showed expression signatures consistent with viral entry, with up-regulation of genes such as PI3K, FAK or JNK. At $48 \mathrm{~h}$ and $96 \mathrm{~h}$, infected cells showed a clear anti-viral response, characterised by the expression of IFNA2 or IRF2.

Conclusions: This study has increased our understanding of the cellular response of Atlantic salmon during ISAV infection, and revealed potential host-virus interactions at the cellular level. The results highlight the value of single-cell sequencing to characterise cell culture models of viral infection, and the results can be exploited in future functional studies to increase the resistance of Atlantic salmon to ISAV.

\section{Keywords: ISAV, Salmo salar, single-cell, RNA-Seq, aquaculture}

\section{Introduction}

Aquaculture is currently the fastest growing food industry worldwide (FAO 2020), and aquaculture products are a fundamental component of healthy and sustainable human diets. Atlantic salmon is the most important aquaculture fish species by value (FAO 2020), and is a high-tech industry underpinned by large research and development programmes. However, infectious diseases remain a major issue for salmon production, such as infectious salmon anaemia (ISA) caused by virulent 
strains of the ISA virus (ISAV). ISAV is an Orthomyxovirus, and is therefore from the same viral family as Influenza viruses. It is a segmented negative stranded RNA virus, and its genome is formed by 8 RNA segments coding for at least 10 proteins (Rimsad and Markussen, 2019). The disease is characterised by severe anaemia, commonly accompanied by haemorrhage and necrosis of various organs (Aamelfot et al, 2014). ISA disease is listed as notifiable by the World Animal Health Organization, and can cause up to $90 \%$ mortality in sea pens. Outbreaks have previously decimated entire national aquaculture industries, as exemplified by the epidemic in Chile (2007-2009, Aamelfot et al, 2014; Godoy et al, 2008). ISAV has been a notifiable pathogen for over 30 years, meaning that upon detection whole stocks have to be culled and the farm is quarantined for a period of time. This culling has major negative impacts on local salmon farms, but also on international trade, exemplified by recent bans on import of genetic material from Norway to the UK.

Preventative and control measures for ISAV include the aforementioned biosecurity measures, and several commercial vaccines also exist. However, these are insufficient to fully control the disease, and improvement of host resistance by selective breeding is an important target. Encouragingly, there is evidence for moderate heritability of resistance to ISA in several studies of Atlantic salmon, and this is being utilised by breeding companies to improve resistance based on regular experimental challenge testing of broodstock families and genomic selection (Kjoglum et al. 2008; Gjerde et al. 2009; Holborn et al. 2020; Gervais et al. 2021). While survival during such challenges is an important target trait, understanding the host-pathogen interactions is important for several reasons. Firstly, it can assist in the identification of functional genetic variants to improve genomic selection accuracy and its persistency across distant relatives. Secondly, it can assist with design of new vaccination, treatment, or management strategies. Thirdly, it can lead to targets for future genome editing studies to potentially develop fully ISA-resistant salmon strains.

Previous studies in Atlantic salmon have reported a notable up-regulation of the innate immune system in response to ISAV (Jorgensen et al. 2008; Lauscher et al. 2011; LeBlanc et al. 2012; Dettleff et al. 2017; Gervais et al. 2021), albeit there is a large degree of tissue specificity in the responses (Valenzuela-Miranda et al. 2015). This is expected since pathogen infections lead to complex and dynamic interactions with the host and its immune system. In vitro models provide simplified systems to study the interactions between pathogens and the host cell machinery, which can help break down the multidimensional responses observed in vivo. Multiple ISAV infection in vitro studies have been performed, describing a rapid interferon response (Andresen et al. 2020; Samsing et al. 2020 ) or the interplay between host and virus over the control of oxidative stress and apoptosis (Olavarria et al. 2015a, b). In vitro studies have also been key to understand the role of the different ISAV proteins and their interaction with cellular mechanisms (Li et al. 2016; Zhang et al. 2017; Thukral et al. 2018; Fredericksen et al. 2019; Toro-Ascuy et al. 2019).

While these approaches have provided important insights into ISAV infections in Atlantic salmon, our understanding of this host-pathogen interaction is still incomplete. One of the main limits of previous studies is the measure of population-level cellular responses with limited resolution. Recent advances in single-cell sequencing allows us to obtain a comprehensive picture of the cellular heterogeneity during infection, and the relationship between viral transcription and host molecular 
responses. Single cell sequencing has yet to be widely applied in Atlantic salmon, but has been used to improve understanding of cellular transcription in gills (West et al. 2021). Here, to better understand the molecular mechanisms of ISAV infection and the Atlantic salmon response to the virus, we have performed an in vitro ISAV infection in the salmon head kidney 1 cell line (SHK-1), which has been widely used to study cellular responses to infection. The results reveal the heterogeneity of the infection process and provide a better understanding of the molecular interactions between ISAV and Atlantic salmon.

\section{Methods}

Cell line

Atlantic salmon head kidney 1 (SHK-1) cells, an immortalised macrophage-like cell line from Atlantic salmon (Salmo salar), was obtained from the European Collection of Authenticated Cell Cultures (ECACC-97111106). All cells were grown as a monolayer in L15 complete media (L15*), L15 (SigmaAldrich, St. Louis, USA) supplemented with $5 \%$ heat-inactivated foetal bovine serum (FBS) (Gibco, Waltham, USA), $40 \mu \mathrm{M} \beta$-mercaptoethanol (Gibco), 100 units $/ \mathrm{mL}$ penicillin and $100 \mu \mathrm{g} / \mathrm{mL}$ streptomycin (Gibco). Cells were cultured in an incubator at $22 \pm 1{ }^{\circ} \mathrm{C}$ without $\mathrm{CO}$. SHK-1 was split $1: 2$ at $80 \%$ confluency with $1 / 3$ conditioned media.

Viral stock

ISAV stock was obtained from Marine Research (Aberdeen, isolate V4782, derived from UK 2008/2009 outbreak). ISAV was passaged once on 1 x T175 SHK-1 cells ( $20 \mathrm{~mL} \mathrm{L15*}$ but with reduced serum (2\%) and the supernatant collected after $50 \%$ cell death, centrifuged, sterilised using a 0.45 $\mu \mathrm{m}$ filter) and stored in $1 \mathrm{~mL}$ aliquots at $-80 \mathrm{C}$ until use. The viral dose was estimated by flow cytometry. To do this, SHK-1 cells were infected with different doses of the virus for $48 \mathrm{hr}$ in L15* with reduced serum (2\%) at $17^{\circ} \mathrm{C}$, followed by antibody staining (ADL anti-ISAv, AquaMab-P10 and anti-mouse-GFP, Invitrogen A21202) and fluorescence quantification (BD Fortessa X20).

\section{ISAV disease challenge}

$2.5 \times 10^{5}$ SHK-1 cells were seeded in 6-well plates in L15 + 10\% FCS and antibiotics (penicillin and streptomycin) $24 \mathrm{~h}$ prior to the challenge experiment. The cells were infected with $200 \mu \mathrm{L}$ of ISAV in L15* with reduced serum (2\%) and incubated at $17^{\circ} \mathrm{C}$ for $24 \mathrm{~h}, 48 \mathrm{~h}$ and $96 \mathrm{~h}$. Cells (three infected timepoints and control) were collected by trypsin treatment, washed in phosphate buffered saline (PBS, Invitrogen) and suspended to 106 cells/mL in PBS + 0.05\% BSA (Bio-Rad cell counter) (Figure 1).

\section{Single-cell RNA sequencing library preparation and sequencing}

Cells were counted and checked for viability on a Cell Counting Slide (Bio-Rad Laboratories Ltd) and then appropriately diluted according to $10 x$ Guidelines. Each individual sample was loaded separately into the 10x Chromium machine and 10x single cell 3' GEM kit v3 was used to generate the libraries following the manufacturer's protocol. The quality of the resulting libraries was assessed in a 
Bioanalyzer (Agilent) and sequenced in two lanes of a NovaSeq SP flowcell with a cycle setup of 28/8/91 at Edinburgh Genomics.

Single-cell RNA sequencing analysis

The raw single-cell RNA-seq samples [0h (pre-infection), 24h, $48 \mathrm{~h}$ and $96 \mathrm{~h}$ post-infection] were demultiplexed and mapped to a combined reference transcriptome of Atlantic salmon (Ensembl, GCA_000233375.4) and ISAV (NCBI, GCF_000854145.) using Alevin/Salmon v1.4.0 (Srivastava et al. 2019). The resulting raw count matrices were analysed using Seurat v3.1.5 (Stuart at al. 2019) in $R$ v3.6.3 (R Core Team 2017). Each library was loaded individually, discarding genes identified in fewer than 3 cells, and cells with fewer than 200 expressed genes. DoubletFinder V2.0.3 was used to assess the impact of doublets on cell clustering in each library, which was negligible. Thereafter all libraries were merged into a single Seurat object. For each cell, the percentage of mitochondrial and viral transcripts were estimated. After inspection of quality control parameters, cells with fewer than 1,000 or more than 10,000 genes or with percentage of mitochondrial transcripts above 25 were discarded. The infected/uninfected status of all cells was calculated as previously described (Sun et al. 2020) based on the kernel density estimate of the distribution of percentage of viral counts on the $\log 10$ scale, which was used to find the first local minima. Cell cycle scores for each cell were estimated using the orthologues of Seurat's (v3.1.5; Stuart at al. 2019) list of mammalian cell cycle markers. The gene counts of the filtered cells were normalised using SCTransform with the percentage of mitochondrial RNA and cell cycle scores as regression variables. Dimensionality reduction was performed using the Uniform Manifold Approximation and Projection (UMAP) method, and clustered in groups using the 'FindNeighbors' and 'FindClusters' functions of Seurat (Stuart at al. 2019). Marker genes for each cell group were determined using the Wilcoxon Rank Sum test ( $\log F C>0.25$ and false discovery rate (FDR) corrected $p$-values $<0.05)$. Kyoto Encyclopedia of Genes and Genomes (KEGG) enrichment analyses were carried out using KOBAS v3.0.3 (Xie et al. 2011). Briefly, salmon genes were annotated against the KEGG protein database (Kanehisa and Goto, 2000) to determine KEGG Orthology (KO). KEGG enrichment for gene lists was tested by comparison to the whole set of expressed genes (obtained from the Seurat object) using Fisher's Exact Test. KEGG pathways with $\geq 5$ DE genes assigned and showing a Benjamini-Hochberg FDR corrected $p$ value $<0.05$ were considered enriched.

\section{Results}

To assess the intracellular activity of ISAV and study the corresponding host response, single-cell RNA-Seq was performed on SHK cells infected with ISAV $24 \mathrm{~h}, 48 \mathrm{~h}$ and $96 \mathrm{~h}$ post infection (Figure 1). Uninfected SHK cells were used as controls. Raw reads were assigned to either Atlantic salmon genes or ISAV genes using a combined host-virus reference transcriptome. A total of 638, 512, 296 and 323 cells passed quality control filters for the control, $24 \mathrm{~h}, 48 \mathrm{~h}$ and $96 \mathrm{~h}$ samples respectively. Clustering of the cells in these four libraries based on the host transcriptional response showed a clear separation between control \& $24 \mathrm{~h}$ infected samples and the $48 \mathrm{~h} \& 96 \mathrm{~h}$ infected samples (Figure 1). A small fraction of the cells showed an unexpected behaviour (i.e. clustering together with cells from a 
different and unexpected timepoint), but this can be attributed to index hopping and crosscontamination with ambient mRNA (Farouni et al. 2020).

Figure 1. Experimental design and cell clustering
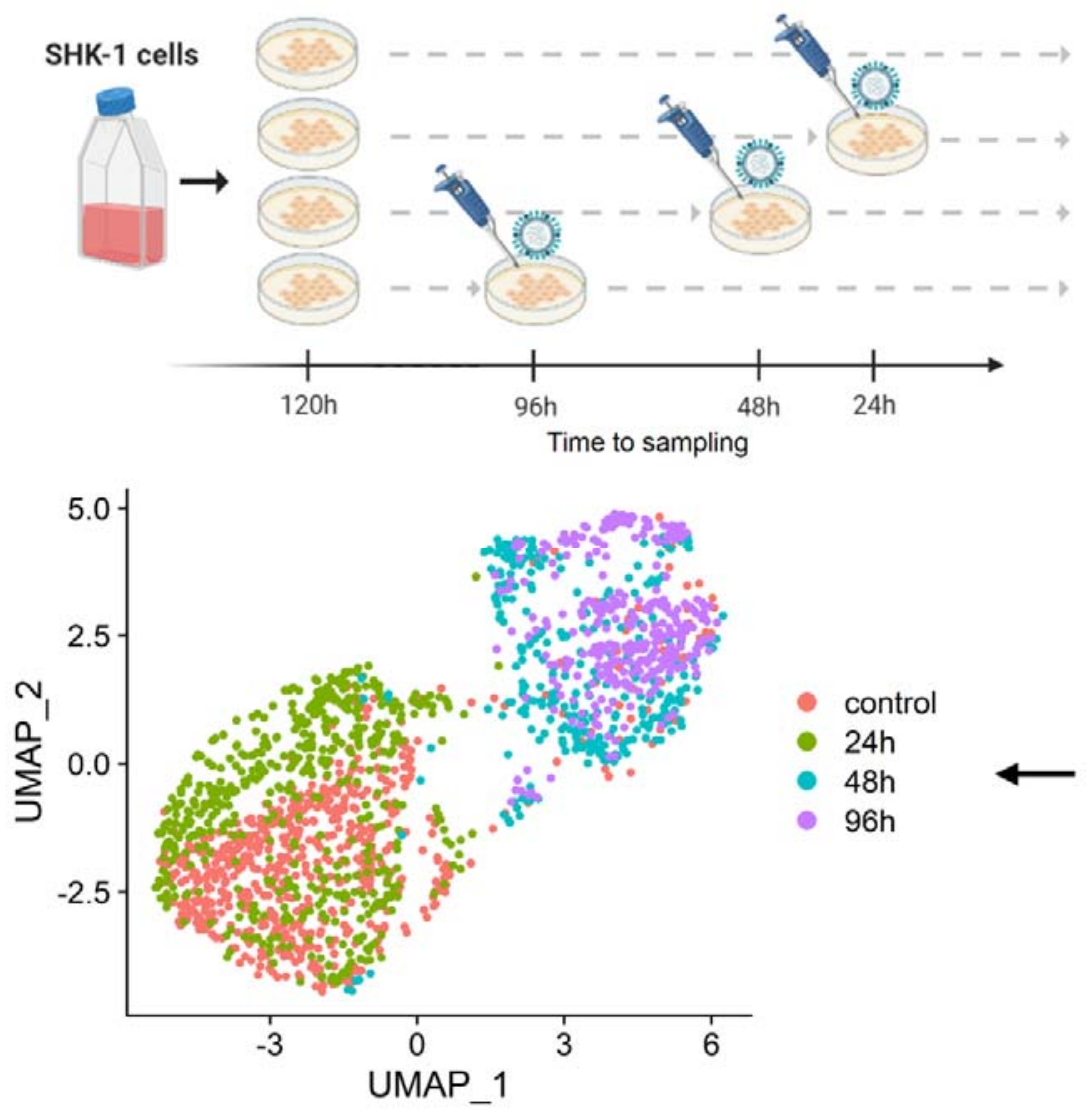

Single-cell library preparation
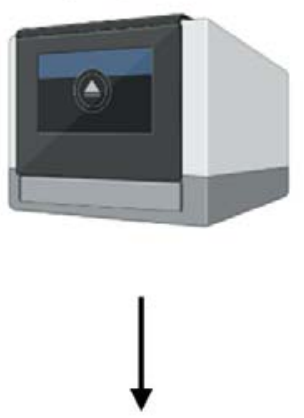

Illumina sequencing

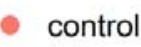

- $24 \mathrm{~h}$

- $48 \mathrm{~h}$

- $96 \mathrm{~h}$

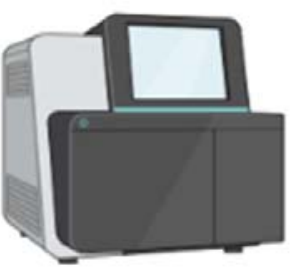

\section{Viral transcription during the infection of Atlantic salmon cells}

The percentage of infected cells (estimated following Sun et al. 2020) was $16 \%$ at $24 \mathrm{~h}$ post-infection, and $32-33 \%$ at 48 and $96 \mathrm{~h}$ (Figure $2 \mathrm{~A}$ ). The infected cells formed two different groups, one with a small number of $24 \mathrm{~h}$ infected cells, and the largest one with $48 \mathrm{~h}$ and $96 \mathrm{~h}$ infected cells, where the percentage of viral transcripts was markedly higher, representing more than $20 \%$ of the transcriptome of the cells (Figure 2B). Expectedly, the percentage of virus transcriptome, relative to the total transcriptome increased over the course of infection, mostly ranging between $2 \%$ and $15 \%$ (Figure $2 \mathrm{C}$ ). This is consistent with previous reports in other orthomyxovirus, although a larger heterogeneity has been described, with viral transcript levels reaching up to $90 \%$ of the cell transcriptome (Russel et al. 2018; Sun et al. 2020). Viral transcript levels can vary substantially depending on the cell type (Sun et al. 2020), but also ISAV infection progression is typically slower than that of other orthomyxovirus, thus, later timepoints could show higher ISAV transcript levels. 
Figure 2. ISAV infection and transcription. A) UMAP dimensionality reduction plot showing the infected and uninfected cells. B) UMAP dimensionality reduction plot showing the viral load of each cell measured as percentage of viral transcripts. C) Viral load in each sample, measured as percentage of viral transcripts. D) Dotplot showing the expression level and the percentage of host cells with transcripts of each viral gene in each sample. E) Heatmap showing the correlation between the expression of the viral genes.

A)

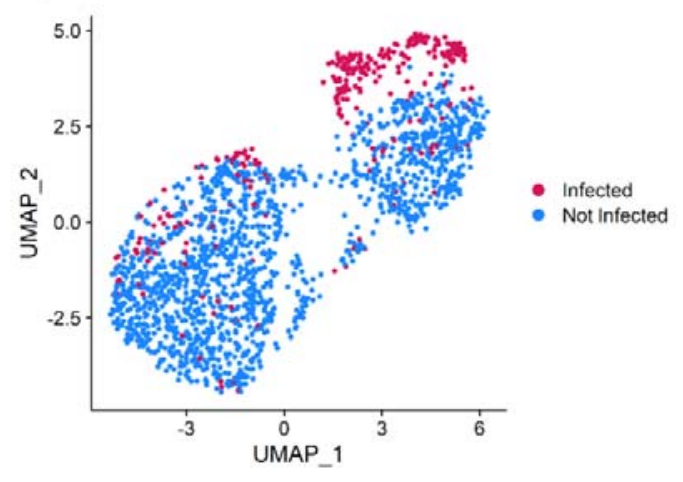

C)

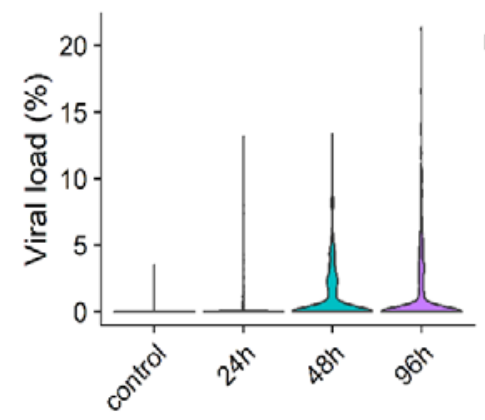

B)

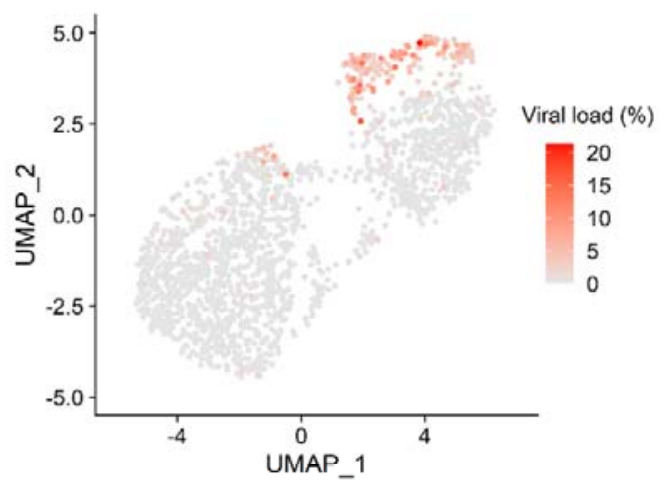

E)

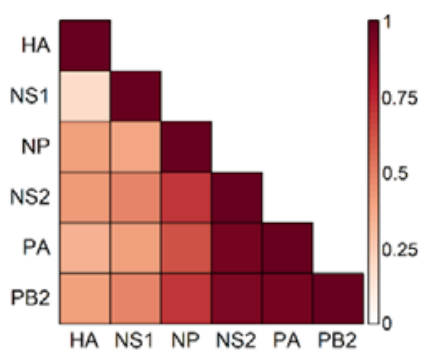

The timing and level of expression of each viral gene also varies along the infection process (Figure 2D); the viral polymerase basic protein 2 (PB2) gene is the most expressed throughout the infection, followed closely by the polymerase acidic protein (PA) and the non-structural proteins 1 (NS1) and 2 (NS2, also termed nuclear exporting protein or NEP). NS1 and NS2 were measured together to avoid the $3^{\prime}$ bias of 10x single-cell RNA-Seq technology; NS2 is a splicing product of NS1 (Ramly et al. 2013) and is expressed at lower levels according to previous reports in ISAV (Ramly et al. 2014) and Influenza A (Robb et al. 2010). The expression of these three genes showed a high positive correlation (Figure 2E), suggesting their co-expression early during the infection. Expression of the hemagglutinin (HA) is found in a small fraction of cells, and it shows the lowest correlation with the other (expressed) viral genes (Figure 2E). Expression of the bicistronic mRNA encoding the matrix protein (M1) and a nuclear export protein (M2, also termed S8ORF2), of the polymerase basic 1 (PB1) and the viral membrane fusion protein (FO) was not detected in our dataset (Figure 2D), which suggests they are either expressed late during the infection or expressed at low levels. In Influenza A 
all the viral mRNAs show similar transcription patterns (Phan et al. 2021), however as mentioned above, ISAV infection typically progresses at a slower rate so it is possible that the viral mRNA dynamics are slightly different and easier to detect.

Host response to ISAV

The cells were clustered in 8 different groups according to the host gene expression (Figure $3 \mathrm{~A}$ ). Clusters 1 to 4 represent the control and 24h samples; most control cells are part of clusters 1,2 and 3 , which also have many $24 \mathrm{~h}$ cells, while cluster 4 is composed mostly of $24 \mathrm{~h}$ cells with an important number of infected cells. Clusters 6 to 8 represent the $48 \mathrm{~h}$ and $96 \mathrm{~h}$ samples; cluster 6 is formed by $48 \mathrm{~h}$ and $96 \mathrm{~h}$ uninfected cells, while cluster 7 and 8 are $48 \mathrm{~h}$ and $96 \mathrm{~h}$ infected cells, respectively. The clear separation of control-24h and $48 \mathrm{~h}-96 \mathrm{~h}$ uninfected cells suggests paracrine regulation by infected cells. Paracrine signalling has been described before in response to Influenza A (Ramos et al. 2019) and other viruses (Patil et al. 2015; Voigt et al. 2016), eliciting an antiviral interferon-mediated response in uninfected cells. Finally, cluster 5 is the most difficult to interpret since it is formed by a low number of cells of each sample apparently not connected to the infection process (Figure 3A).

Each of these clusters is characterised by specific marker genes (Figure 3B, Supplementary File). Clusters 1 and 2 are characterised by the high expression of certain ribosomal genes, which also show a high expression in half of the cells of group 3. This suggests manipulation of the ribosomal machinery by ISAV, which can be connected to the hijacking of host translational machinery by the virus, a common strategy used by virus to repress the cellular mRNA translation and allow the preferential translation of viral mRNAs (Levene and Gaglia, 2018). On the other hand, a group of immune genes, including interferon alpha 2 (Ifna2) or interferon regulatory factor 2 (Irf2), are highly expressed in infected cells at $48 \mathrm{~h}$ (cluster 7), and moderately in infected cells at 96h (cluster 8), suggesting either viral repression or a shift in the immune response later during the infection. Further, this cluster is not highly expressed in non-infected cells at $48 \mathrm{~h}$ and $96 \mathrm{~h}$ (cluster 6), suggesting that paracrine signalling by infected cells does not promote the activation of an interferon response in bystander cells.

KEGG pathway analysis (Figure 3 C) confirmed the enrichment in ribosomal genes in the uninfected cells clusters from early timepoints ( 1 and 2). Clusters 3 and 4 (mostly $24 \mathrm{~h}$ cells, especially cluster 4 ) are enriched for "focal adhesion" and "ECM-receptor interactions", likely a consequence of the interaction of ISAV with the extracellular matrix and membrane receptors, and successive internalization. The genes in these pathways include phosphoinositide 3-kinase (PI3K), focal adhesion kinase (FAK) or c-Jun terminal kinase (JNK), important for Influenza A entry into the cytoplasm (Ayllon et al. 2012; Elbanesh et al. 2014; Zhang et al. 2018). This is consistent with the similarity in the entry mechanisms of Influenza A and ISAV (Eliassen et al. 2000; Kibenge and Kibenge, 2016) and suggests that molecular interactions during viral entry are also conserved to some extent. These early genes are good candidates for functional studies aimed at disrupting ISAV entry into salmon cells. 
Uninfected cells at $48 \mathrm{~h}$ and $96 \mathrm{~h}$ (cluster 6 ) showed a non-specific state of alert, with terms such as "proteasome", "RNA degradation", "mRNA surveillance pathway" or "cytosolic DNA-sensing pathway". For instance, this cluster shows expression of the seven Ism genes that compose the Lsm17 ring, involved in the degradation of messenger RNA in the cytoplasm (REF). Many proteasomespecific genes are also expressed in this cluster, including two of the subunits of the immunoproteasome, involved in the degradation of intracellular proteins, including those of viral origin (REF). Connected to the proteasome, this cluster also expresses all the genes of the ubiquitination machinery: E1 ubiquitin-activating enzymes, E2 ubiquitin-conjugating enzymes and E3 ligases of all the types (HERC3, U-box and RING-finger and their adaptor proteins). Spliceosome genes are also enriched, suggestive of active post-transcriptional and post-translational mechanisms in this cluster.

Uninfected cells at $48 \mathrm{~h}$ and $96 \mathrm{~h}$ (cluster 6 ) also showed enrichment in ribosomal genes, as uninfected control and 24h cells (cluster 1 and 2), which was unexpected based on the expression pattern of ribosomal genes in the heatmap (Figure 3B). However, we found that the underlying specific ribosomal genes are different, with only two genes in common between the two groups of cells (out of 55 in each list). These two sets of ribosomal genes show contrasting patterns of expression (Figure 3D ), with the "control" ribosomal genes showing less expression in infected cells, while the "infected" ribosomal genes show increased expression at $48 \mathrm{~h}$ and $96 \mathrm{~h}$. A closer look the gene lists revealed that most of the "infected" ribosomal genes code for proteins of the mitochondrial ribosome (Supplementary file $\mathrm{X}$ ). Mitochondria activate anti-viral immune responses through mitochondrial antiviral signalling (MAVS; Refolo et al. 2020) and can also initiate apoptosis (Lei et al. 2009). Influenza A NS1 protein has been observed in the mitochondria (Tsai et al. 2017), and is able to alter mitochondria morphodynamics (Pila-Castellanos et al. 2021). Our results suggests that paracrine regulation upon ISAV infection activates the mitochondria as a defence mechanism, and the fact that these mitochondria ribosomal genes are also overexpressed at $96 \mathrm{~h}$ in infected cells (Figure 3D, cluster 8) suggests that they play a role in the immune response against ISAV.

Finally, clusters 7 and 8 (infected $48 \mathrm{~h}$ and $96 \mathrm{~h}$ cells) show up-regulation of key immune response genes, such as interferon alpha 2 and 3 (ifna2, ifna3), interferon regulatory factor 2 (irf2) or C-X-C motif chemokine 10 (cxl10). These genes are slightly more up-regulated in the $48 \mathrm{~h}$ cells group (Figure 4), with pathways such as toll-like receptor signalling, RIG-I receptor signalling, TNF signalling or cytokine-cytokine interaction. However, these genes were not expressed in uninfected cells at 48 and 96h (cluster 6). IFNA2, IFNA3 and CXL10 are secreted proteins that act on other cells to promote an antiviral state and could explain the "activated" state of cluster 6 cells. However, surprisingly they would not induce the up regulation of interferon genes. 
Figure 3. Response to ISAV in the Atlantic salmon SHK-1 cell line. A) UMAP dimensionality reduction plot showing the clustering of the cells according to the host cell transcriptome; B) Heatmap showing the expression pattern of top marker genes of the UMAP cluster; C) Barplot with the fold enrichment of selected KEGG pathways in each cell cluster; D) Ridgeplot showing the proportion of the transcriptome that represent the genes assigned to the enriched KEGG pathway "Ribosome" in cluster 1 and cluster 5.

A)

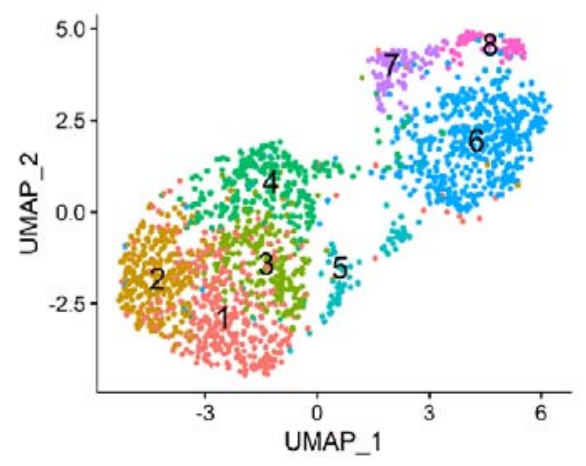

B)

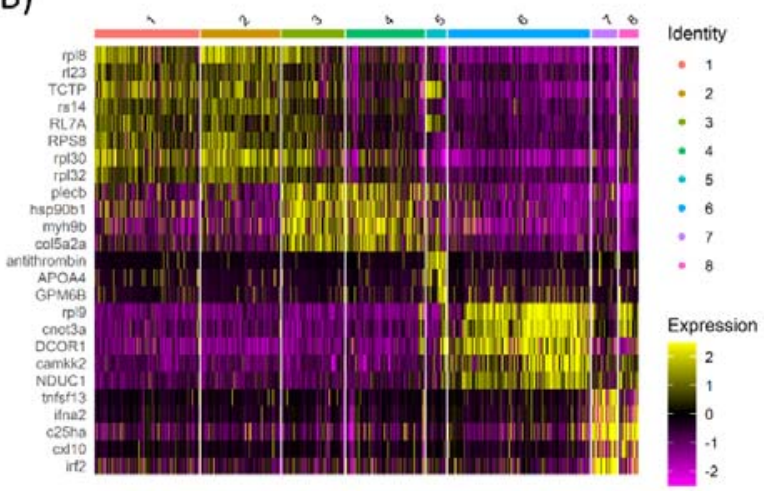

C)

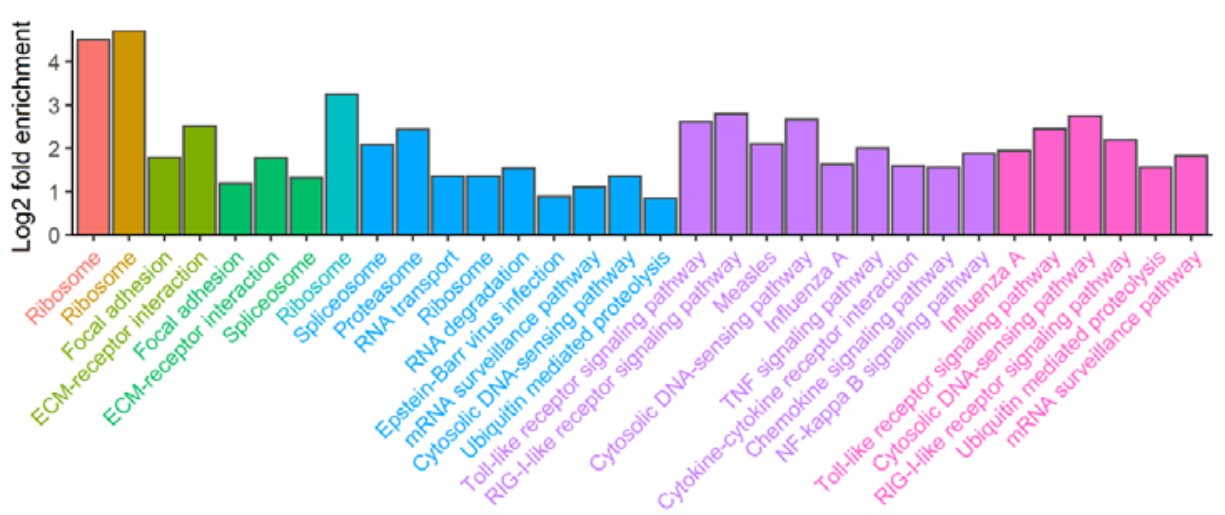

D)

Ribosome genes (control)

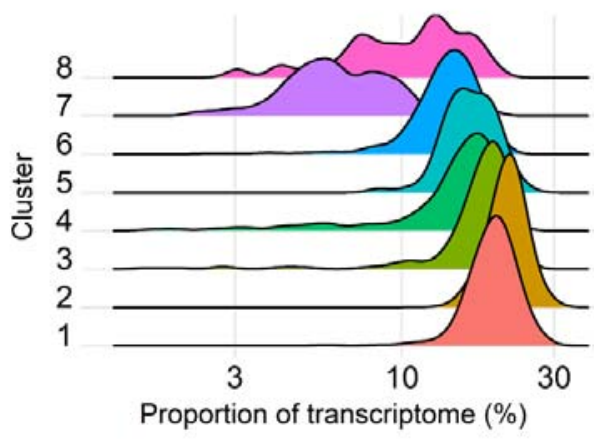

Ribosome genes (infected)

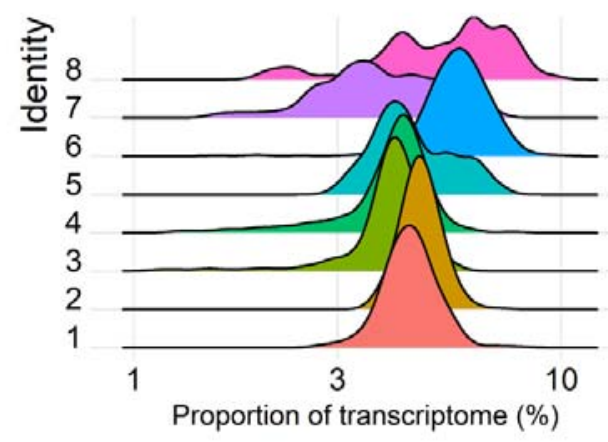




\section{Interactions between host and viral transcripts}

To evaluate whether specific viral proteins could have a direct impact on the expression of host genes, a correlation analysis between viral and host genes was performed for infected cells (Figure 4, Supplementary file). PB2, PA and NS1+NS2 expression patterns showed significant correlation with largely the same set of host genes $(r>|0.3|)$, as expected considering the highly correlated expression of these viral genes. Considering these viral genes are the most expressed, these correlations do not necessarily represent regulation of host genes by viral proteins, they could also represent genes that respond proportionally to viral load. In fact, the host genes exhibiting the highest positive correlation include the immune genes mentioned in the previous section (ifna2, ifna3, irf2) and the regulator of antiviral signalling probable ATP-dependant RNA helicase DHX58 (dhx58). The highest correlation was observed with cholesterol 25-hydroxylasel A (c25ha), an interferon-stimulated gene involved in the regulation of cholesterol biosynthesis, which has broad antiviral activity (Liu et al. 2013). However, these viral genes, especially NS1+NS2, also show negative correlations with several transcription factors, such as twist-related protein 2 (twst2) or zinc-finger protein SNAl1 (snai1).

Figure 4. Correlation between viral gene expression and host gene expression

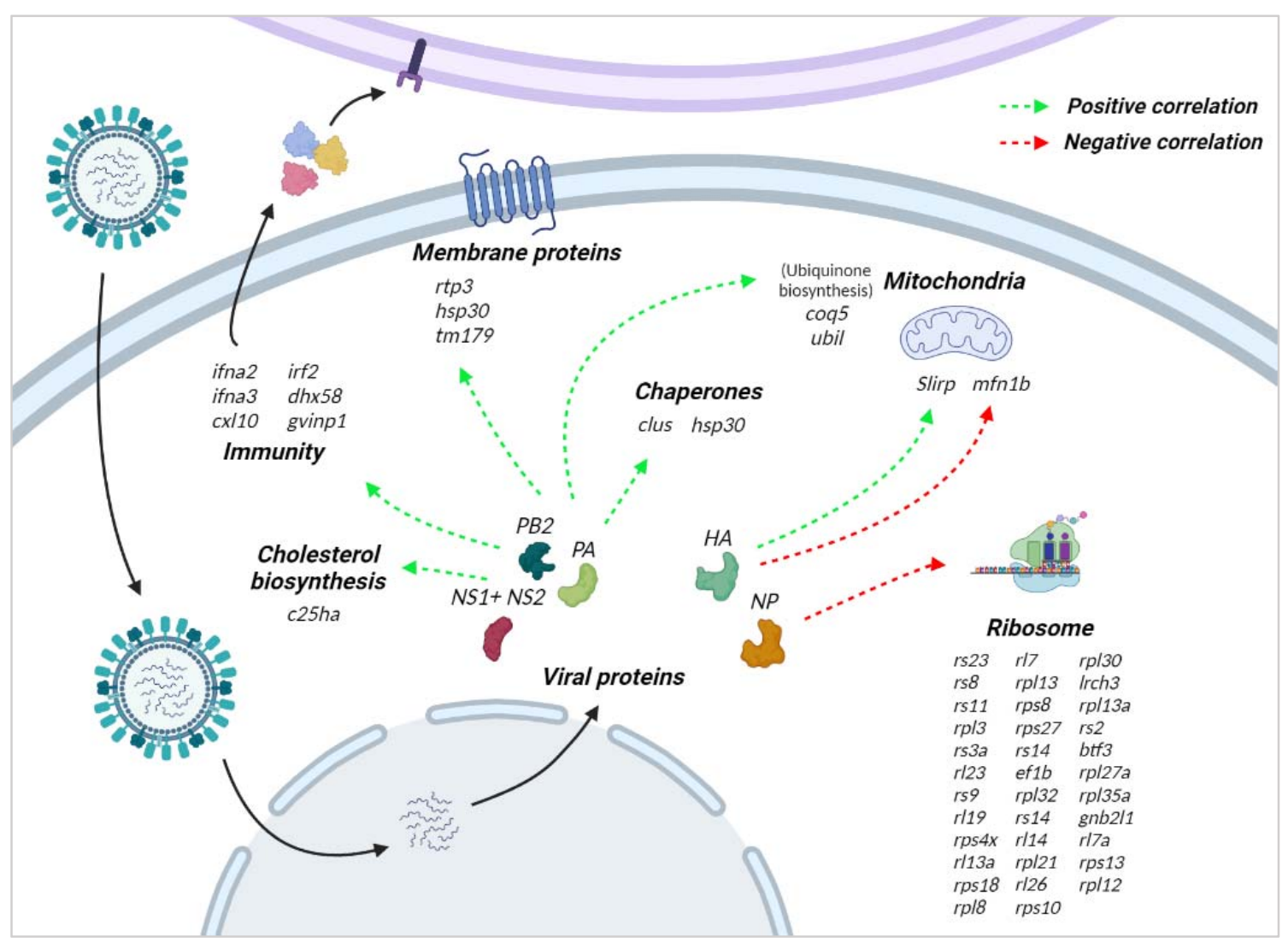


Consistent with previous results, the expression of over thirty cytoplasmic ribosomal genes is negatively correlated with the expression of the viral nucleoprotein NP $(r=-0,3$ to -0.4) (Figure 5), suggesting a negative regulation of the host translation machinery by the virus. While NP is a structural protein, it has been shown that it interacts with host factors to facilitate viral replication (Momose et al. 2001; Kawaguchi et al. 2011), however no interactions with ribosomal genes have been previously described. There were also several chaperons showing positive correlation with viral genes, such as clusterin (clu), which has antiapoptotic activity and is directly targeted by the Influenza A virus nucleoprotein (Tripathi et al. 2013). Another protein of interest is sequestosome-1 (sqstm1), a receptor required for selected autophagy that inhibits Seneca valley virus and avian influenza replication (Wen et al. 2021; Liu et al. 2021).

\section{Discussion}

In this study single-cell RNA sequencing was applied to study ISAV infection in Atlantic salmon SHK-1 cells. This simplified system, in combination with this novel genomics technology, has allowed us to finely characterise the transcriptomic changes that occur during ISAV infection both in the virus and the host, and suggest potential interactions. While cell line models are often used to study hostpathogen interaction, this study highlights that even within an immortalised cell line there is major heterogeneity in both infection status and cellular immune response. Our study complements and expands knowledge gained in previous in vivo and in vitro studies of ISAV infections in Atlantic salmon.

Our results align with previous studies that have shown that ISAV triggers an early immune response characterised by the activation of interferon genes, both in vitro and in vivo (Jorgensen et al. 2007; Svingerud et al. 2013; Valenzuela-Miranda et al. 2015). An interesting finding is that the interferon response was only triggered in infected but not in bystander cells, which is quite different to what has been reported during Influenza infections, where the paracrine signalling is important at $12 \mathrm{~h}$ post infection, increasing the expression of interferon stimulated genes in bystander cells (Ramos et al. 2019). There are different potential explanations for this finding, for instance the virus may be able to inhibit the interferon-related paracrine signalling quite early during the infection, but it is also plausible that paracrine signalling functions differently in fish and mammals. Further investigations are necessary to understand fish paracrine signalling, including studies with other species and viruses.

The ribosomal machinery is an important part of viral replication since viral genomes do not usually harbour mRNA translation genes, and therefore they need to recruit host proteins including ribosomal proteins (RPs) to complete their replication cycle. Our result shave shown that the viral protein NP may interact or at least drive the expression of specific mitochondrial RPs. While no interactions between NP and ribosomal proteins have been described, it is well known that viruses interact with ribosomal proteins as part of their infection strategy (Li 2019; Dong et al. 2020). However, recent studies have also highlighted that some ribosomal proteins can have an antiviral function by either interacting with viral proteins to inhibit transcription/translation or by activating 
antiviral defence signalling pathways (Li 2019). Non-infected cells in $48 \mathrm{~h}$ and $96 \mathrm{~h}$ infected samples showed a clear up-regulation of mitochondrial RPs, which could suggest that they form an important part of an antiviral state pathway in bystander cells, potentially repressed by the viral protein NP in infected cells considering the negative correlation.

The proteasome and ubiquitination also seem to be an important component of the antiviral state in bystander cells. We have also found up-regulation of genes related to ubiquitination in resistant fish in response to ISAV (manuscript in preparation). Moreover, the protein encoded by ISAV segment 8, which is known to interfere with interferon signalling, can be conjugated to ubiquitin and the ubiquitin-like interferon simulated gene 15 (ISG15) is Atlantic salmon cells (Olsen et al. 2016). Several viruses can hijack the host ubiquitination machinery, and in particular Influenza uses host ubiquitination as part of its cell entry and replication strategy (Rudnicka and Yamauchi, 2016; Huang et al. 2018; Gu and Fada, 2020).

Our study has highlighted significant correlations between viral and host genes, including several chaperones. Chaperones are used by viruses to facilitate the folding and assembly of the viral proteins (Aviner and Frydman, 2022). There are also host genes associated to autophagy process that are correlated with viral genes. Previous studies have reported that mammalian cells can use autophagy to restrict the replication of avian Influenza (Liu et al. 2021), but it has also been reported that it can promote the replication of influenza A (Wang et al. 2019; Zhang et al. 2021). In any case, autophagy-related genes and process may represent novel targets for the development of anti-ISAV therapeutics.

\section{Conclusion}

Early ISAV infection of Atlantic salmon SHK cells is characterised by high expression of the PB2, NS1+NS2 and PA viral genes, which show highly correlated expression patterns. After $24 \mathrm{~h}$ of infection infected SHK cells showed similar transcriptomic profiles to control cells, however genes and pathways connected to viral entry were identified, which could be good targets for functional studies to impair viral infection. At $48 \mathrm{~h}$ and $96 \mathrm{~h}$ post-infection there was a clear host transcriptomic response to the virus which also affected uninfected cells, therefore suggesting paracrine signalling from infected cells. These paracrine signalling seemed to produce an unspecific alert state in the uninfected cells, involving mRNA surveillance and RNA degradation, ubiquitination and the proteasome, or mitochondrial activation. These pathways were also up-regulated in $48 \mathrm{~h}$ and $96 \mathrm{~h}$ infected cells, but they also showed a clear antiviral interferon response, including genes such as ifna or irf2. Correlation between the expression of viral and host genes suggested potential negative regulation of certain host transcription factors and ribosomal genes by viral proteins. This study increases our understanding of the molecular interactions between ISAV and Atlantic salmon cells, and provides new targets for functional studies aiming to increase the resistance of Atlantic salmon stocks, leading to increased food security and fish welfare. 


\section{Acknowledgements}

This work was supported by an RCUK-CONICYT grant (BB/N024044/1) and BBSRC Institute Strategic Funding Grants to the Roslin Institute (BBS/E/D/20002172, BBS/E/D/30002275, and BBS/E/D/10002070). Sequencing was carried out by Edinburgh Genomics, which is partly supported through core grants from NERC (R8/H10/56), MRC (MR/K001744/1), and BBSRC (BB/J004243/1).

\section{References}

Aamelfot M, Dale OB, Falk K. 2014. Infectious salmon anaemia - pathogenesis and tropism. Journal of Fish Diseases, 37:291-307.

Andresen AMS, Boudinot P, Gjoen T. 2020. Kinetics of transcriptional response against poly (I:C) and infectious salmon anaemia virus (ISAV) in Atlantic salmon kidney (ASK) cell line. Developmental and Comparative Immunology, 110:103716.

Aviner R, Frydman J. 2022. Proteostasis in Viral Infection: Unfolding the Complex Virus-Chaperone Interplay. Additional Perspectives on Protein Homeostasis. Cold Spring Harbour Perspectives Biology.

Ayllon J, García-Sastre A, Hale BG. 2012. Influenza A viruses and PI3K. Virulence, 3:411-414.

Dettleff P, Moen T, Santi N, Martinez V. 2017. Transcriptomic analysis of spleen infected with infectious salmon anemia virus reveals distinct pattern of viral replication on resistant and susceptible Atlantic salmon (Salmo salar). Fish Shellfish Immunology, 61:187-93.

Dong HJ, Zhang R, Kuang Y, Wang XJ. 2020. Selective regulation in ribosome biogenesis and protein production for efficient viral translation. Archives of Microbiology, 203:1021-1032.

Elbahesh H, Cline T, Baranovich T, Govorkova EA, Shultz-Cherry S, et al. 2014. Novel Roles of Focal Adhesion Kinase in Cytoplasmic Entry and Replication of Influenza A Viruses. Journal of Virology, 88:6714-6728.

Eliassen TM, Froystad MK, Dannevig BH, Jankowska M, Brech A, et al. 2000. Initial Events in Infectious Salmon Anemia Virus Infection: Evidence for the Requirement of a Low-pH Step. Journal of Virology, $74: 218-227$.

FAO. 2020. The State of World Fisheries and Aquaculture 2020. Sustainability in action. Rome.

Farouni R, Djambazian H, Ferri LE, Raoussis J, Najafabadi HS. 2020. Model-based analysis of sample index hopping reveals its widespread artifacts in multiplexed single-cell RNA-sequencing. Nature Communications, 11:2704.

Fredericksen F, Villalba M, Maldonado N, Payne G, Torres F, et al. Sumoylation of nucleoprotein (NP) mediated by activation of NADPH oxidase complex is a consequence of oxidative cellular stress 
during infection by Infectious salmon anemia (ISA) virus necessary to viral progeny. Virology, 531:269-279.

Gervais O, Barria A, Papadopoulou A, Gratacap RL, Hillestad B, et al. 2021. Exploring genetic resistance to infectious salmon anaemia virus in Atlantic salmon by genome-wide association and RNA sequencing. BMC Genomics, 22:345.

Gjerde B, Evensen O, Bentsen HB, Storset A. 2009. Genetic (co)variation of vaccine injuries and innate resistance to furunculosis (Aeromonas salmonicida) and infectious salmon anaemia (ISA) in Atlantic salmon (Salmo salar). Aquaculture, 287:52-58.

Godoy MG, Aedo A, Kibenge MJT, Groman DB, Yason CV, et al. 2008. First detection, isolation and molecular characterization of infectious salmon anaemia virus associated with clinical disease in farmed Atlantic salmon (Salmo salar) in Chile. BMC Veterinary Research, 4:28.

Gu H, Fada BJ. 2020. Specificity in Ubiquitination Triggered by Virus Infection. International Journal of Molecular Sciences, 21:4088.

Holborn MK, Ang KP, Elliott JAK, Powell F, Boulding EG. 2020. Genome wide analysis of infectious salmon anemia resistance in commercial Saint John river Atlantic salmon. Aquaculture, 514:734514.

Huang X, Wei S, Ni S, Huang Y, Qin Q. 2018. Ubiquitin-proteasome system is required for efficient replication of Singapore grouper iridovirus. Frontiers in Microbiology, 9:2798.

Jørgensen SM, Afanasyev S, Krasnov A. 2008. Gene expression analyses in Atlantic salmon challenged with infectious salmon anemia virus reveal differences between individuals with early, intermediate and late mortality. BMC Genomics, 9:179.

Jorgensen SV, Hetland DL, Press CMcL, Grimholt U, Gjoen T. 2007. Effect of early infectious salmon anaemia virus (ISAV) infection on expression of MHC pathway genes and type I and II interferon in Atlantic salmon (Salmo salar L.) tissues. Fish and Shellfish Immunology, 23:576-588.

Kanehisa M, Goto S. 2000. KEGG: kyoto encyclopedia of genes and genomes. Nucleic Acids Research, 28:27-30.

Kawaguchi A, Momose F, Nagata K. 2011. Replication-coupled and host factor-mediated encapsidation of the influenza virus genome by viral nucleoprotein. Journal of Virology, 85:61976204.

Kibenge FSB, Kibenge MJT. 2016. Orthomyxoviruses of Fish. Aquaculture Virology, 2016:299-326.

Kjoglum S, Henryon M, Aasmundstad T, Korsgaard I. 2008. Selective breeding can increase resistance of Atlantic salmon to furunculosis, infectious salmon anaemia and infectious pancreatic necrosis. Aquaculture Research, 39:498-505.

Lauscher A, Krossøy B, Frost P, Grove S, König M, et al. 2011. Immune responses in Atlantic salmon (Salmo salar) following protective vaccination against infectious salmon anemia (ISA) and subsequent ISA virus infection. Vaccine, 29:6392-401. 
LeBlanc F, Arseneau JR, Leadbeater S, Glebe B, Laflamme M, et al. 2012. Transcriptional response of Atlantic salmon (Salmo salar) after primary versus secondary exposure to infectious salmon anemia virus (ISAV). Molecular Immunology, 51:197-209.

Lei Y, Moore CB, Liesman RM, O'Connor BP, Bergstralh DT, et al. 2009. MAVS Mediated Apoptosis and Its Inhibition by Viral Proteins. PloS One, 4:e5466.

Levene RE, Gaglia MM. 2018. Host Shutoff in Influenza A Virus: Many Means to an End. Viruses, 10:475.

Li C, Greiner-Tollersrud L, Robertsen B. 2016. Infectious salmon anemia virus segment 7 ORF1 and segment 8 ORF2 proteins inhibit IRF mediated activation of the Atlantic salmon IFNa1 promoter. Fish and Shellfish Immunology, 52:258-262.

Li S. 2019. Regulation of Ribosomal Proteins on Viral Infection. Cells, 8:508.

Liu S, Mok BW, Deng S, Liu H, Wang P, et al. 2021. Mammalian cells use the autophagy process to restrict avian influenza virus replication. Cell Reports, 35:109213.

Liu SY, Aliyari R, Chikere K, Li G, Marsden MD, et al. 2013. Interferon-inducible cholesterol-25hydroxylase broadly inhibits viral entry by production of 25-hydroxycholesterol. Immunity, 38:92105.

Momose F, Basler CF, O'Neill RE, Iwamatsu A, Palese P, et al. 2001. Cellular splicing factor RAF2p48/NPI-5/BAT1/UAP56 interacts with the influenza virus nucleoprotein and enhances viral RNA synthesis. Journal of Virology, 75:1899-1908.

Olavarría VH, Recabarren P, Fredericksen F, Villalba M, Yáñez A. 2015. ISAV infection promotes apoptosis of SHK-1 cells through a ROS/p38 MAPK/Bad signalling pathway. Molecular Immunology, 64:1-8.

Olavarría VH, Valdivia S, Salas B, Villalba M, Sandoval R, et al. 2015. ISA virus regulates the generation of reactive oxygen species and p47phox expression in a p38 MAPK-dependent manner in Salmo salar. Molecular Immunology, 63:227-234.

Olsen CM, Markussen T, Thiede B, Rimstad E. 2016. Infectious Salmon Anaemia Virus (ISAV) RNA binding protein encoded by segment 8 ORF2 and its interaction with ISAV and intracellular proteins. Viruses, 8:52.

Patil S, Fribourg M, Ge Y, Batish M, Tyagi S, et al. 2015. Single-cell analysis shows that paracrine signaling by first responder cells shapes the interferon- $\beta$ response to viral infection. Science Signaling, 8:363.

Phan T, Fay EJ, Lee Z, Aron S, Hu WS, et al. 2021. Segment-Specific Kinetics of mRNA, cRNA, and vRNA Accumulation during Influenza Virus Infection. Virology, 95:e02102-e02120. 
Pila-Castellanos I, Molino D, McKellar J, Lines L, da Graca J, et al. 2021. Mitochondrial morphodynamics alteration induced by influenza virus infection as a new antiviral strategy. PLoS Pathogens, 17:e1009340.

R Core Team (2017). R: A language and environment for statistical computing. R Foundation for Statistical Computing. Vienna, Austria. URL https://www.R-project.org/.

Ramly RB, Olsen CM, Braaen S, Hansen EF, Rimstad E. 2014. Transcriptional regulation of gene expression of infectious salmon anaemia virus segment 7. Virus Research, 190:69-74.

Ramly RB, Olsen CM, Braaen S, Rimstad E. 2013. Infectious salmon anaemia virus nuclear export protein is encoded by a spliced gene product of genomic segment 7. Virus Research, 177:1-10.

Ramos I, Smith G, Ruf-Zamojski F, Martinez-Romero C, Fribourg M, et al. 2019. Innate Immune Response to Influenza Virus at Single-Cell Resolution in Human Epithelial Cells Revealed Paracrine Induction of Interferon Lambda 1. Journal of Virology, 93:e00559-00619.

Refolo G, Vescovo T, Piacentini M, Fimia GM, Ciccosanti F. 2020. Mitochondrial Interactome: A Focus on Antiviral Signaling Pathways. Frontiers in Cell Developmental Biology, 8:8.

Rimstad E, Markussen T. 2019. Infectious salmon anaemia virus - molecular biology and pathogenesis of the infection. Journal of Applied Microbiology, 129:85-97.

Rudnicka A, Yamauchi Y. 2016. Ubiquitin in Influenza Virus Entry and Innate Immunity. Viruses, 8:293.

Russell AB, Trapnell C, Bloom JD. 2018. Extreme heterogeneity of influenza virus infection in single cells. eLife, 7:e32303.

Samsing F, Hoad J, Mohr P, Dearnley M, Wynne JW. 2020. Comparative transcriptome analysis of pilchard orthomyxovirus (POMV) and infectious salmon anaemia virus (ISAV). Fish and Shellfish Immunology, 105:415-426.

Srivastava A, Malik L, Smith T, Sudbery I, Patro R. 2019. Alevin efficiently estimates accurate gene abundances from dscRNA-seq data. Genome Biology, 20:65.

Stuart T, Butler A, Hoffman P, Hafemeister C, Papalexi E, et al. 2019. Comprehensive integration of single-cell data. Cell, 177:1888-1902.

Sun J, Vera JC, Drnevich J, Lin YT, Ke R, Brooke CB. 2020. Single cell heterogeneity in influenza A virus gene expression shapes the innate antiviral response to infection. PLoS Pathogens, 16:e1008671.

Svingerud T, Holand JK, Robertsen B. 2013. Infectious salmon anemia virus (ISAV) replication is transiently inhibited by Atlantic salmon type I interferon in cell culture. Virus Research, 177:163-170.

Thukral V, Varshney B, Ramly RB, Ponia SS, Mishra SK, et al. 2018. s8ORF2 protein of infectious salmon anaemia virus is a RNA-silencing suppressor and interacts with Salmon salar Mov10 (SsMov10) of the host RNAi machinery. Virus Genes, 54:199-214. 
Toro-Ascuy D, Santibañez A, Peña V, Beltran-Pavez C, Cottet L, et al. 2019. Development of an Isavirus minigenome system to study the function of the pocket RNA-binding domain of the viral nucleoprotein (NP) in salmon cells. Journal of Fish Diseases, 43:197-206.

Tripathi S, Batra J, Sharma K, Patel JR, Ranjan P, et al. 2013. Influenza A virus nucleoprotein induces apoptosis in human airway epithelial cells: implications of a novel interaction between nucleoprotein and host protein Clusterin. Cell Death and Disease, 4:e562.

Tsai CF, Lin HY, Hsu WL, Tsai CH. 2017. The novel mitochondria localization of influenza A virus NS1 visualized by FIAsH labelling. FEBS Open Biology, 7:1960-1971.

Valenzuela-Miranda D, Boltaña S, Cabrejos ME, Yáñez JM, Gallardo-Escárate C (2015) Highthroughput transcriptome analysis of ISAV-infected Atlantic salmon Salmo salar unravels divergent immune responses associated to head-kidney, liver and gills tissues. Fish Shellfish Immunology, 45:367-377.

Voigt EA, Swick A, Yin J. 2016. Rapid induction and persistence of paracrine-induced cellular antiviral states arrest viral infection spread in A549 cells. Virology, 496:59-66.

Wang R, Zhu Y, Zhao J, Ren C, Li P, et al. 2019. Autophagy Promotes Replication of Influenza A Virus In Vitro. Journal of Virology, 93:e01984-02018.

Wen W, Li X, Yin M, Wang H, Qin L, et al. 2021. Selective autophagy receptor SQSTM1/ p62 inhibits Seneca Valley virus replication by targeting viral VP1 and VP3. Autophagy, 17:3763-3775.

West AC, Mizoro Y, Wood SH, Ince LM, Iversen M, et al. 2021. Immunologic Profiling of the Atlantic Salmon Gill by Single Nuclei Transcriptomics. Frontiers in Immunology, 12:669889.

Xie C, Mao X, Huang J, Ding Y, Wu J, et al. 2011. KOBAS 2.0: a web server for annotation and identification of enriched pathways and diseases. Nucleic Acids Research, 39:W316-322.

Zhang J, Ruan T, Sheng T, Wang J, Sun J, et al. 2019. Role of c-Jun terminal kinase (JNK) activation in influenza A virus-induced autophagy and replication. Virology, 526:1-12.

Zhang RH, Zhang HL, Li PY, Li CH, Gao JP, et al. 2021. Autophagy is involved in the replication of H9N2 influenza virus via the regulation of oxidative stress in alveolar epithelial cells. Virology Journal, $18: 22$

Zhang W, Cai C, Lin L, Tao YJ, Jin M. 2017. Subcellular localization and interactions of Infectious Salmon Anemia Virus (ISAV) M1 and NEP as well as host Hsc70. Virology Journal, 14:30. 